\title{
Componentes funcionais da memória visuoespacial
}

CESAR GALERA, RICARDO BASSO GARCIA - RAFAEL VASQUES

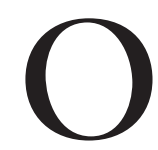

CONCEITO de memória de trabalho refere-se ao sistema de armazenamento temporário e de processamento de informações envolvido nas diversas atividades cognitivas complexas que realizamos. De acordo com seu modelo mais utilizado, a memória de trabalho é formada por um processador com capacidade de atenção limitada, o executivo central, que controla três subsistemas armazenadores. Informações de natureza verbal são armazenadas pelo laço fonológico, um sistema duplo formado por um armazenador passivo baseado em códigos acústico-fonológicos e por um sistema ativo de recitação que impede o decaimento desses códigos. Informações referentes aos objetos e às relações espaciais entre eles são armazenadas pelo esboço visuoespacial, também considerado um sistema duplo formado por um armazenador visual e por um sistema ativo, responsável por manter informações visuoespaciais (Logie, 1995). Por fim, o buffer episódico proporciona uma interface entre os armazenadores verbal e visuoespacial, integrando e armazenando em representações complexas as informações provenientes dos subsistemas e da memória de longo prazo (Baddeley, 2000; 2007).

A divisão da memória de trabalho em subcomponentes especializados baseia-se em evidências empíricas que incluem déficits específicos em pacientes com lesões cerebrais (Della Sala; Logie, 1999), por diferentes taxas de desenvolvimento da memória verbal e visuoespacial observadas em estudos com crianças (Hitch, 1990; Logie; Pearson, 1997) e pelos efeitos de interferência seletiva no armazenamento de informações de diferentes modalidades (Baddeley, 1986; Logie, 1995). Estudos centrados na dissociação dupla também foram importantes na fragmentação da memória de trabalho. Esse método permite supor que uma dada função A é separada ou dissociada de uma função B se existirem pessoas com deficiências na função A mas função B preservada, e pessoas com a função A intacta e deficiências na função B (Shallice, 1988). Por exemplo, De Renzi e Nichelli (1975) identificaram dois grupos de pacientes com lesões cerebrais, um grupo com déficit de memória verbal e memória espacial preservada, e o outro grupo com o padrão inverso, memória verbal intacta e déficit de memória espacial. Essa dissociação dupla, confirmada em outros estudos (por exemplo, Basso et al., 1982; Hanley et al. 1991; Logie, 1986), suporta a subdivisão entre dois sistemas de armazenamento temporário, um verbal e outro visuoespacial. 
Em estudos de laboratório centrados em pessoas saudáveis, dissociações funcionais são obtidas por procedimentos de tarefas duplas; isto é, ao memorizar um conjunto de informações, o participante realiza uma outra tarefa concorrente. A lógica subjacente é que se ambas as tarefas partilham recursos cognitivos, então o armazenamento de informações é prejudicado, ou seja, podem-se simular os efeitos de uma lesão cerebral através da sobrecarga de uma certa função cognitiva (Baddeley, 2007; 2012). Entretanto, se as tarefas não partilham recursos, o desempenho dos participantes é semelhante ao observado quando as tarefas são realizadas separadamente.

Por meio de tarefas duplas, portanto, é possível identificar os tipos de sobrecarga cognitiva que influenciam uma dada tarefa de memória. Apresentamos como esse método permitiu uma melhor compreensão do armazenamento temporário de informações visuoespaciais.

\section{O esboço visuoespacial}

Ao longo do desenvolvimento do modelo de memória de trabalho, o componente visuoespacial foi menos estudado que o fonológico (Baddeley, 2007; Pearson, 2001). Nos últimos anos, esse panorama se modificou com o crescente interesse nesse tema, dada sua relevância teórica e aplicada. Processos visuoespaciais são cruciais em diversas atividades cotidianas e estão sujeitos a variabilidade entre indivíduos e a deficiências específicas, seja durante o desenvolvimento, seja em processos de envelhecimento patológico (Cornoldi; Vecchi, 2003), podendo até mesmo contribuir para um diagnóstico precoce da doença de Alzheimer (Della Sala et al., 2012; Parra et al., 2009).

Inicialmente, poucas considerações foram feitas acerca da estrutura do esboço visuoespacial, concebido em termos gerais como um sistema de memorização temporária de informações e de criação e manutenção de imagens mentais (Baddeley, 1986). Essa abrangência se refletiu na variedade de procedimentos experimentais, cada qual priorizando um desses aspectos de seu funcionamento. Nos últimos anos, pode-se notar uma convergência para procedimentos experimentais mais específicos, que consideram o acúmulo de evidências sobre a dissociação entre um sistema visual e outro espacial de armazenamento, trazendo importantes implicações teóricas e metodológicas.

\section{A dissociação entre memória espacial e visual}

A tarefa de imaginação de Brooks (1967) foi fundamental para investigar o esboço visuoespacial. Na versão espacial dessa tarefa, o participante deve imaginar uma matriz de dimensão 4 x 4 e memorizar uma sequência de frases com orientações espaciais ("no quadrado inicial coloque 1", "no quadrado à direita coloque 2", "no quadrado abaixo coloque 3", e assim por diante). Na versão verbal, o participante deve memorizar frases semelhantes, mas sem conotação espacial ("no quadrado inicial coloque 1", "no quadrado bom coloque 2", "no quadrado lento coloque 3", e assim por diante). Após ouvir uma sequência 
de frases, o participante deve repeti-las na ordem em que foram apresentadas. Como se pode supor, a versão espacial é mais fácil do que a verbal, pois o participante pode imaginar uma trajetória na matriz e "ler" essa imagem mental para repetir as sentenças.

Em situações de tarefa dupla, nas quais a tarefa de Brooks foi aliada a outros tipos de tarefa, foi observado que o desempenho dos participantes na versão espacial é prejudicado pela execução de uma tarefa de rastreamento motor na qual o participante persegue um alvo em movimento (Baddeley et al., 1975), mesmo que esteja de olhos vendados (Baddeley; Lieberman, 1980). Por sua vez, a versão verbal não é afetada pelo rastreamento do alvo. Além disso, ambas as versões da tarefa de Brooks não foram afetadas por uma tarefa de natureza visual que exigia a discriminação de brilho. Tais resultados sugerem, em primeiro lugar, uma sobreposição entre os recursos cognitivos necessários para manter a informação visuoespacial e aqueles necessários para realizar a tarefa de rastreamento. Em segundo lugar, a versão espacial e a tarefa de rastreamento utilizam os recursos de um sistema de processamento espacial, mas não de um sistema visual, o que seria esperado dado que exige a formação de uma imagem mental. Em vista disso, Baddeley e Lieberman (1980) argumentaram que o esboço visuoespacial opera sobre representações de natureza espacial.

O padrão de interferência observado por Baddeley e Lieberman (1980) pode ter resultado do aspecto espacial da tarefa de Brooks, baseada na imaginação de uma trajetória. Segundo Logie (1986), se os participantes realizassem uma tarefa de imaginação visual, o desempenho seria prejudicado por uma tarefa visual concorrente. De fato, a memorização de listas de palavras por meio de uma estratégia mnemônica visual é prejudicada pela observação de estímulos visuais irrelevantes, mas não é afetada por estímulos verbais irrelevantes. Quando a estratégia de memorização é verbal, baseada na simples repetição dos itens da lista, o desempenho é prejudicado por estímulos verbais, mas não por estímulos visuais. Em conjunto, esses resultados sugerem um sistema visuoespacial composto por dois subcomponentes, um dinâmico, dedicado à informação espacial, e outro passivo, dedicado à informação visual (Logie, 1995; 2011).

Evidências robustas apontam que essa subdivisão também ocorre em tarefas de memória nas quais, diferente das tarefas de imaginação, o participante memoriza um estímulo apresentado visualmente. Tarefas concorrentes de observação passiva ou de discriminação visual interferem na memória para características visuais como a cor e a forma dos estímulos, e tarefas espaciais ou de movimento interferem na memória para localizações (Darling et al., 2007; 2009; Darling et al., 2006; Klauer; Zhao, 2004; Tresch et al., 1993).

Em vista dessa dissociação, diferentes procedimentos experimentais são empregados para estudar a memória visual e espacial. Um dos principais instrumentos para se estudar a memória espacial é a tarefa de blocos de Corsi (1972) ou uma de suas variantes (Berch et al., 1998), em que estímulos visualmente 
iguais variam de posição no espaço. A memória visual é estudada por meio de tarefas que envolvem padrões visuais como matrizes de células pretas e brancas, letras em diferentes fontes tipográficas, cores, figuras ou faces, em geral estímulos visualmente distintos, com baixa demanda de processamento espacial e elaborados para dificultar o uso de estratégias verbais (cf. Avons, 1998; Darling et al., 2009; Della Sala et al., 1999; Smyth et al., 2005).

Não é, contudo, consensual a definição de quais características dos estímulos são visuais e quais são espaciais. Para alguns autores, o termo visual refere-se à aparência de um objeto ou de uma cena, suas cores, formas, contrastes, tamanho e textura, assim como a localização relativa entre objetos de acordo com uma perspectiva estática. Por sua vez, o termo espacial refere-se a aspectos que são dinâmicos, como trajetórias e posições espaciais apresentadas em sequência, assim como movimentos de um local a outro em uma cena (Della Sala; Logie, 2002; Logie, 1995; 2011). Outros autores apontam para uma maior complexidade das representações espaciais, e argumentam pela distinção entre espacial-sequencial e espacial-simultâneo (Cornoldi; Vecchi, 2003; Pazzaglia; Cornoldi, 1999), ou ainda pela distinção entre espacial categórico (posições codificadas em relação ao observador) e espacial configuracional (posições relativas entre objetos visuais) (Liesefeld; Zimmer, 2011).

Em vista desse contexto, especial atenção deve ser dada às características dos estímulos, aos procedimentos de apresentação de estímulos e à escolha de tarefas concorrentes adequadas. Para se ter uma ideia dos aspectos metodológicos que devem ser observados, apresentamos abaixo uma síntese dos principais fatores relacionados à subdivisão visual/espacial da memória de trabalho.

\section{Memória visual}

Evidências sugerem que a memória de trabalho opera com códigos de natureza visual. Em particular, dois fenômenos corroboram essa perspectiva: o efeito de similaridade visual e o efeito de interferência causado por imagens irrelevantes ou por ruídos visuais. É mais difícil armazenar estímulos visualmente similares do que estímulos dissimilares, sugerindo que a sobreposição de traços visuais interfere na recuperação dos itens na memória (Logie; Van der Meulen, 2009). Esse efeito é análogo ao de similaridade fonológica observado na memória verbal, em que é mais difícil lembrar uma sequência de letras com sons semelhantes (b, p, d, v) do que letras com sonoridade diferente (c, j, k, m) (Conrad, 1964; Baddeley, 1986; 1997).

O efeito de similaridade visual surge de forma mais evidente quando o estímulo utilizado é de difícil nomeação ou quando os participantes são impedidos de utilizarem estratégias verbais de memorização mediante uma tarefa concorrente verbal, na qual a repetição de uma sílaba irrelevante, "la, la, la, ...", impede a verbalização dos estímulos visuais para auxiliar a tarefa de memória. Por exemplo, Hue e Erickson (1988) mostraram que a memória para ideogramas chineses depende tanto da frequência de uso dos ideogramas (pouco utilizados 
vs. muito utilizados) como da similaridade entre eles. Para os ideogramas mais frequentes, os participantes utilizavam uma estratégia predominantemente verbal, não sendo observado efeito de similaridade visual ou interferência de uma tarefa concorrente visual. Por sua vez, efeitos de similaridade foram observados para ideogramas de baixa frequência, ou seja, quando o acesso a representações fonológicas era mais lento. Resultados análogos foram observados por Hitch et al. (1988) em um estudo da memória para desenhos de objetos do cotidiano, com nomes curtos e longos, em crianças de cinco e de dez anos de idade. As crianças mais novas são suscetíveis à similaridade visual, mas as crianças mais velhas, não. Essas recordavam um número menor de figuras com nomes mais longos, sugerindo que os mais jovens utilizam um código visual e os mais velhos, um fonológico.

Embora afetando um sistema funcionalmente independente, a similaridade visual influencia também na manutenção da informação fonológica. Isso é evidente no estudo de Logie et al. (2000) em que a recordação de palavras escritas visualmente similares (exemplo, FLY, PLY, DRY...) é mais difícil do que pouco similares (SIGH, LIE, PI...). Isso pode explicar por que pessoas com déficits severos de memória verbal podem ter uma capacidade de memória para dígitos praticamente normal quando os dígitos são apresentados na forma escrita. No caso desse efeito da similaridade visual sobre a recordação de estímulos verbais, a similaridade visual pode se restringir apenas aos últimos estímulos apresentados para memorização. No entanto, em estímulos que não permitem codificação verbal, o efeito da similaridade pode se estender por toda a lista memorizada (Galera; Fuhs, 2003).

O efeito da informação irrelevante ocorre especialmente em tarefas de memória que utilizam imagens mentais visuais, como em técnicas mnemônicas em que imagens são geradas internamente a partir de instruções verbais. Em uma dessas técnicas, conhecida como peg-word, o participante aprende associar imagens mentais previamente aprendidas às palavras de uma lista que deve memorizar. Depois, recorda as palavras lembrando-se das imagens associadas. Em outra técnica, conhecida desde os gregos como o método dos locais, o participante lembra-se de um ambiente real, bem conhecido, e distribui nesse ambiente as palavras ou conceitos que quer memorizar. Depois, quando deve recordar essa informação, percorre mentalmente os lugares em que foram depositadas.

Assim como a memória fonológica é prejudicada pela fala irrelevante vinda do ambiente (Salamé; Baddeley, 1982), a recordação de material verbal memorizado com uso de imagens visuais também tem se mostrado sensível à informação irrelevante. Pois se as imagens mentais e a percepção visual partilham os mesmos recursos cognitivos, então a apresentação de informação visual irrelevante deve interferir na criação e manutenção de imagens visuais. De fato, no estudo de Logie (1986) a apresentação de figuras irrelevantes interferiu na recordação de uma lista de palavras quando o participante utiliza a técnica do peg-word. Outra 
técnica de interferência, o Ruído Visual Dinâmico (RVD), foi criada por Quinn e McConnell (1996; 2006; McConnell; Quinn, 2000; 2004). O RVD consiste em apresentar um ruído semelhante ao produzido por uma tela de TV fora de sintonia enquanto o participante gera as imagens visuais das palavras a serem memorizadas, ou imagina os locais em que as imagens das palavras serão colocadas.

A interferência do RVD é consistente em tarefas que utilizam a geração de imagens visuais e tem efeitos exclusivamente sobre o material processado visualmente (McConnell; Quinn, 2004; Quinn; McConnell, 1996; Toms et al., 1994). O RVD e técnicas semelhantes afetam a imaginação de rotas a serem percorridas durante uma escalada (Smyth; Waller, 1998), o tempo de comparação de imagens geradas a partir da memória de longo prazo (Dean et al., 2005; Parker; Dagnall, 2009) e a vivacidade ou nitidez de uma imagem visual (Baddeley; Andrade, 2000). O RVD afeta o processo de criação das imagens mentais visuais enquanto os participantes as estão formando a partir de instruções verbais, ou quando o participante deve recuperá-las durante a recordação, mas não quando é apresentado durante o intervalo que antecede a recordação. Ou seja, o RVD parece afetar a imagem visual enquanto ela se encontra em um armazenador visual acessível conscientemente (Quinn; McConnel, 2006), embora ainda não tenha sido descartado o argumento de que o RVD afeta processos de atenção envolvidos na geração e na recuperação das imagens (Andrade et al., 2002; Logie, 2003).

Em tarefas de memória, nas quais os estímulos são apresentados visualmente, sem que seja necessário imaginá-los, o efeito do RVD é inconsistente. Por exemplo, o RVD não tem efeitos significativos no reconhecimento de caracteres chineses (Andrade et al., 2002) ou de matrizes preenchidas aleatoriamente (Avons; Sestieri, 2005), embora possa afetar a confiança que os participantes têm sobre a acurácia de suas respostas (Kemps; Andrade, 2012). Por outro lado, o RVD afetou o reconhecimento para o tamanho exato de ć́rculos (McConnell; Quinn, 2004), para tons de cores (Dent, 2010; Pereira; Galera [em preparo]), detalhes de textura (Dean et al., 2008) e de fontes gráficas (Darling et al., 2007; 2009; Santana et al. [no prelo]). Essa diferença no efeito do RVD em tarefas de memória com uso de imagens mentais visuais e em tarefas com uso de estímulos visuais pode ser explicada se funções diferentes estiverem envolvidas em cada tipo de tarefas (Borst et al., 2012; Logie; Van der Meulen, 2009).

A diferença, contudo, do efeito do RVD em tarefas de memória com estímulos mais complexos como caracteres chineses ou padrões geométricos (Andrade et al., 2002; Avons; Sestieri, 2005) e aquelas que dependem da recordação de detalhes de tamanho, cor ou forma (Darling et al., 2007; 2009; Dent, 2010; McConnell; Quinn, 2004; Pereira; Galera [em preparo]; Santana et al. [no prelo]) ainda não foi esclarecida. De acordo com Quinn (2012), essa diferença sugere que sistemas diferentes podem estar envolvidos no armazenamento dos dois tipos de estímulos. Estímulos mais complexos, como caracteres chineses e 
padrões visuais, seriam codificados mais facilmente em termos semânticos, enquanto estímulos definidos por variações sutis no tamanho, cor ou forma permaneceriam mais tempo em um armazenador visual pré-semântico, talvez mais suscetível à interferência da informação sensorial. Se considerarmos que o RVD atua em um nível sensorial, sem ativar representações de longo prazo, seu efeito pode ser considerado uma evidência de que a representação visual afetada pelo RVD permanece em um armazenador visual pré-categórico, anterior à interpretação semântica, tal como proposto por Kosslyn (1994), Pearson (2001) e Quinn $(2008 ; 2012)$, mas não por Logie (2011). Trata-se de uma problemática fundamental para a compreensão da arquitetura da memória de trabalho, que deve englobar os sistemas responsáveis pela geração de imagens mentais, pelo registro de detalhes visuais e pelo armazenamento temporário que é sensível à similaridade visual.

\section{Memória espacial}

Além da tarefa de Brooks (1967), uma das tarefas comportamentais mais utilizadas para avaliar memória espacial é o Teste de Blocos de Corsi (TBC; Corsi, 1972), realizado com um tabuleiro no qual estão distribuídos de forma irregular nove blocos de igual dimensão. Durante a avaliação, o examinador aponta uma sequência de blocos, a uma taxa de um bloco por segundo e, após o término da sequência, o participante deve apontar os blocos na mesma ordem em que foram apresentados. O número de blocos de uma sequência aumenta progressivamente ao longo do teste até que o participante não consiga recordar a sequência inteira. Assim, a capacidade da memória espacial de um participante é definida pela extensão da maior sequência recordada corretamente (De Renzi; Nichelli, 1975; Orsini et al., 2001).

A capacidade da memória espacial conforme medida pelo TBC ou testes semelhantes, além de ser limitada pela quantidade de blocos, também é afetada por características próprias das sequências. A recordação de sequências longas ou que contenham cruzamentos é mais difícil do que de sequências curtas ou sem cruzamentos (Galera; Souza, 2010; Orsini et al., 2001). Nos estudos de Kemps (2001) a capacidade de recordação de sequências espaciais foi determinada pela redundância de cada sequência. A proximidade temporal ou espacial das posições facilita a recordação de sequências (Farrand et al., 2001; Parmentier et al., 2006). Rossi-Arnaud et al. (2006) sugerem que o executivo central pode estar envolvido tanto na codificação como no armazenamento das sequências espaciais, independentemente de essas sequências serem redundantes ou não. Se a vantagem da assimetria não depende inteiramente da atividade do esboço visuoespacial nem do executivo central, onde a simetria é codificada? De acordo com esses autores a simetria seria uma contribuição da memória de longo prazo, que seria integrada à informação da memória visuoespacial.

Assim como a tarefa espacial de Brooks é afetada por tarefas concorrentes que exigem a realização de movimentos, o TBC também é afetado pela execução 
de movimentos. Quinn e Ralston (1986) mostraram que o desempenho nessa tarefa é prejudicado quando o participante deve realizar uma tarefa de dedilhar um teclado que não pode ver. Outros estudos mostraram que a recordação das sequências de blocos é prejudicada pela execução de movimentos das mãos e braços (Smyth; Pendleton, 1989). No entanto, Quinn (1994) sugere que o prejuízo na tarefa de memória espacial pode não ser o resultado da realização do movimento em si, mas da atenção espacial envolvida na realização do movimento. Essa interpretação é evidente nos estudos de Smyth (1996; Smyth; Scholey, 1994) que mostram que o desempenho na tarefa de Corsi é prejudicado por uma tarefa de monitoramento espacial de sons em que não se exige a realização de movimento, mas sim o deslocamento da atenção pelo espaço. Segundo Awh et al. (1998), a manutenção da informação espacial é efetuada por meio de deslocamentos do foco da atenção para as localizações memorizadas. Mais recentemente Postle et al. (2006) apresentaram evidências que sugerem que o esboço visuoespacial dispõe de um sistema de recitação relacionado aos mecanismos de atenção que controlam os movimentos oculares.

Apesar de serem funcionalmente independentes, não se pode negar uma relação simbiótica entre a localização espacial e a identidade dos objetos, seja ela visual ou verbal. Na tarefa utilizada para investigar a memória da localização de objetos pode-se perguntar ao participante tanto pelo objeto que estava em um determinado local como pelo local em que um dado objeto se encontrava. Estudos que utilizam tarefas de localização de objetos tem mostrado que a representação memorizada é uma representação integrada dos dois tipos de informação: quando o participante deve memorizar o objeto, memoriza também o local em que ele foi visto (Olson; Marshuetz, 2005). Nós encontramos resultados semelhantes, em que a conjunção entre localização e identidade do objeto é incidental, ou seja, acontece de forma involuntária, independentemente das instruções dadas ao participante. Esse tipo de conjunção entre identidade e localização espacial depende da natureza do objeto, se esse é definido em termos visuais ou verbais, e da dimensão utilizada para recuperar a informação memorizada (Corder et al., 2012; Galera; Santana [em submissão]). Além da identidade, a ordem na qual os estímulos são apresentados também é codificada de forma incidental, e isso acontece de forma diferente se o participante deve recordar a identidade ou a localização dos estímulos (Santana; Galera [no prelo]).

\section{Considerações finais}

Apresentamos algumas evidências da existência de sistemas funcionalmente independentes para o armazenamento de curto prazo da informação visual e espacial. Nossa compreensão do funcionamento integrado desses sistemas de memória para objeto e posição se deve, em grande parte, ao modelo de memória de trabalho visuoespacial proposto por Logie (1995). Esse modelo, inspirado parcialmente na arquitetura do laço fonológico (Baddeley, 1986), considera que a memória de trabalho visuoespacial é um sistema duplo, formado por um 
armazenador visual passivo (visual cache) e por um armazenador espacial ativo (inner scribe). A informação vinda do ambiente ativa representações na memória de longo prazo e essa representação é encaminhada a um ou outro armazenador em função de suas características. Especificamente, o armazenador visual passivo é responsável por armazenar o conteúdo visual já interpretado semanticamente. Seu conteúdo pode ser gerado a partir da interpretação de informações visuais, de descrições verbais ou de informações táteis. Ou seja, o armazenador visual passivo não é, na realidade, um armazenador visual, mas um armazenador de representações ativadas da memória de longo prazo.

O armazenador espacial ativo é responsável pela recitação do material contido no armazenador visual passivo e pelo armazenamento de sequências de movimentos dirigidos a posições específicas no espaço, tal como exigidas na tarefa de blocos de Corsi (Logie, 2011). Além de ser responsável por planejar e armazenar movimentos e localizações espaciais, esse componente tem, como já vimos, a função de recitar a informação mantida no armazenador visual. A recitação de traços visuais por um processo de natureza espacial é um aspecto fundamental do modelo que ainda não foi suficientemente detalhado, especialmente quando se levam em conta as evidências de que tarefas espaciais concorrentes não interferem na manutenção de informações visuais, assim como tarefas visuais não interferem na memorização de informações espaciais (Darling et al., 2006; Della Sala et al., 1999; Logie; Marchetti, 1991), tornando menos plausível que a recitação de um sistema visual seja feita por um sistema espacial (Quinn, 2012; Repovs; Baddeley, 2006).

Outro aspecto difícil do modelo de memória de trabalho visuoespacial é considerar que a informação sensorial não tem acesso direto ao visual cache (Logie, 1995; 2011). Traçando um paralelo entre os armazenadores fonológico e visuoespacial, a questão do acesso da informação verbal ao armazenador fonológico tem sido importante para a compreensão da memória de trabalho verbal. Desde o estudo inicial de Salamé e Baddeley (1982), o efeito da fala irrelevante sobre o armazenamento da informação fonológica tem beneficiado o desenvolvimento teórico e fornecido um impulso adicional para reavaliação do papel desse subsistema da memória de trabalho (Jones et al., 2006; 2007). No entanto, para Logie $(1995 ; 2011)$, a informação ambiental é interpretada com base no conhecimento de longo prazo e então transferida para o visual cache, o que o descaracteriza como um armazenador visual. Embora a questão esteja ainda aberta ao debate, as evidências experimentais existentes sugerem que a informação visual vinda do ambiente interfere na geração de imagens mentais, sugerindo um acesso direto ao sistema de armazenamento visual e/ou ao sistema no qual são geradas as imagens mentais (Borst et al., 2012; Dean et al., 2008; Dent, 2010; McConnell; Quinn, 2000; 2004; Quinn; McConnell, 1996; 2006).

A título de conclusão, a literatura apresentada sugere que o armazenamento da informação visuoespacial é realizado por dois sistemas funcionalmente 
independentes, um dedicado ao armazenamento da informação visual e outro à informação espacial. No entanto, a forma como esses sistemas interagem ainda não é clara. Nos últimos anos a memória visuoespacial tem sido beneficiada por um grande volume de estudos, mas continuam existindo muitas áreas de ignorância residual a serem preenchidas pela pesquisa empírica e teórica. Nós apontamos de maneira mais detalhada uma área que nos parece importante, que é a existência mesmo de uma memória visual não interpretada semanticamente. $\mathrm{O}$ modelo de memória de trabalho visuoespacial proposto por Logie (1995; 2011), embora de inegável valor heurístico, deixa em aberto algumas questões, entre elas a de considerar que a memória visuoespacial é acessada apenas por informações interpretadas semanticamente. Do nosso ponto de vista, essa suposição diminui, se não elimina, a importância do componente visual e não se sustenta no modelo de memória de trabalho visuoespacial.

\section{Referências}

ANDRADE, J. et al. Insensitivity of visual short-term memory to irrelevant visual information. The Quarterly Journal of Experimental Psychology, v.55, n.3, p.753-74, 2002.

AVONS, S. E. Serial report and item recognition of novel visual patterns. British Journal of Psychology, v.89, n.2, p.285-308, 1998.

AVONS, S. E.; SESTIERI, C. Dynamic visual noise: No interference with visual short-term memory or the construction of visual images. European Journal of Cognitive Psychology, v.17, n.3, p.405-24, 2005.

AWH, E.; JONIDES, J.; REUTER-LORENZ, P. A. Rehearsal in spatial working memory. Journal of Experimental Psychology: Human Perception and Performance, v.24, n.3, p.780-90, 1998.

BADDELEY, A. D. et al. Imagery and visual working memory. In: RABBIT, P. M. A.; DORNIC, S. (Ed.) Attention and Performance V. London: Academic Press, 1975. p.205-17.

BADDELEY, A. D. Working Memory. London: Oxford University Press, 1986. p.304. . Human Memory: Theory and Practice. Hove: Psychology Press, 1997. p.423. The episodic buffer: a new component of working memory? Trends in Cognitive Sciences, v.4, n.11, p.417-23, 2000. 2007. p.432.

Working Memory, Thought, and Action. New York: Oxford University Press,

Working Memory: Theories, Models, and Controversies. Annual Review of Psychology, v.63, p.1-29, 2012.

BADDELEY, A. D.; ANDRADE, J. Working memory and the vividness of imagery. Journal of Experimental Psychology: General, v.129, n.1, p.126-45, 2000.

BADDELEY, A. D.; LIEBERMAN, K. Spatial Working Memory. In: NICKERSON, R. S. (Ed.) Attention and Performance VIII. Hillsdale: Lawrence Erlbaum Associates Inc, 1980. p.521-39. 
BASSO, A. et al. Left hemisphere damage and selective impairment of auditory verbal short-term memory. A case study. Neuropsychologia, v.20, n.3, p.263-74, 1982.

BERCH, D. B.; KRIKORIAN, R.; HUHA, E. M. The Corsi block-tapping task: Methodological and theoretical considerations. Brain and Cognition, v.38, n.3, p.317-38, 1998.

BORST, G.; NIVEN, E.; LOGIE, R. H. Visual mental image generation does not overlap with visual short-term memory: A dual-task interference study. Memory and Cognition, v.40, n.3, p.360-372, 2012.

BROOKS, L. R. The supression of visualization by reading. The Quarterly Journal of Experimental Psychology, v.19, n.4, p.289-99, 1967.

BUSCH, R. M. et al. Corsi block-tapping task performance as a function of path configuration. Journal of Clinical and Experimental Neuropsychology, v.27, n.1, p.127-34, 2005.

CONRAD, R. Acoustic confusions in immediate memory. British Journal of Psychology, v.55, n.1, p.75-84, 1964.

CORDER, A. P. U. et al. A integração incidental da informação visual e espacial na memória de trabalho. Psicologia: Teoria e Prática, v.14, n.1, p.113-25, 2012.

CORNOLDI, C.; VECCHI, T. Visuo-Spatial Working Memory and Individual Differences. Hove: Psychology Press, 2003. p.169.

CORSI, P. M. Human memory and the medial temporal region of the brain. S. 1.: McGill University, 1972.

DARLING, S. et al. Neuropsychological evidence for separating components of visuospatial working memory. Journal of Neurology, v.253, n.2, p.176-80, 2006.

DARLING, S.; DELLA SALA, S.; LOGIE, R. H. Behavioural evidence for separating components within visuo-spatial working memory. Cognitive Processing, v.8, n.3, p.175-81, 2007.

Dissociation between appearance and location within visuo-spatial working memory. The Quarterly Journal of Experimental Psychology, v.62, n.3, p.417-25, 2009.

DE RENZI, E.; NICHELLI, P. Verbal and non-verbal short-term memory impairment following hemispheric damage. Cortex: A Journal Devoted to the Study of the Nervous System and Behavior, v.11, n.4, p.341-54, 1975.

DEAN, G. M. et al. Selective interference with the use of visual images in the symbolic distance paradigm. Journal of Experimental Psychology: Learning, Memory and Cognition, v.31, n.5, p.1043-68, 2005.

DEAN, G. M.; DEWHURST, S. A.; WHITTAKER, A. Dynamic visual noise interferes with storage in visual working memory. Experimental Psychology, v.55, n.4, p.283-9, 2008.

DELLA SALA, S. et al. Pattern span: a tool for unwelding visuo-spatial memory. Neuropsychologia, v.37, n.10, p.1189-99, 1999.

DELLA SALA, S.; LOGIE, R. H. Neuropsychological impairments of visual and spatial working memory. In: BADDELEY, A. D.; KOPELMAN, M. D.; WILSON, B. A. (Ed.) The Handbook of Memory Disorders. 2.ed. Chichester: John Wiley \& Sons, 2002 . p.271-92.

DELLA SALA, S.; PARRA, M. A.; FABI, K.; LUZZI, S.; ABRAHAMS, S. Short-term 
memory binding is impaired in $\mathrm{AD}$ but not in non-AD dementias. Neuropsychologia, v.50, n.5, p. 833-840, 2012.

DENT, K. Dynamic visual noise affects visual short-term memory for surface color, but not spatial location. Experimental Psychology, v.57, n.1, p.17-26, 2010.

FARRAND, P.; PARMENTIER, F. B. R.; JONES, D. M. Temporal-spatial memory: retrieval of spatial information does not reduce recency. Acta Psychologica, v.106, n.3, p.285-301, 2001.

GALERA, C.; FUHS, C. C. L. Memória visuo-espacial a curto prazo: os efeitos da supressão articulatória e de uma tarefa aritmética. Psicologia: Reflexão e Crítica, v.16, p.337-48, 2003.

GALERA, C.; SANTANA, J. J. R. A. DE. Visual-spatial and verbal-spatial incidental binding in working memory. [em submissão].

GALERA, C.; SOUZA, A. L. P. DE. Memória visuoespacial e cinestésica de curto prazo em crianças de 7 a 10 anos. Estudos de Psicologia, v.15, p.137-43, 2010.

HANLEY, J. R.; YOUNG, A. W.; PEARSON, N. A. Impairment of the visuo-spatial sketch pad. The Quarterly Journal of Experimental Psychology, v.43, n.1, p.101-25, 1991.

HITCH, G. J. et al. Visual working memory in young children. Memory and Cognition, v.16, n.2, p.120-32, 1988.

HITCH, G. J. Developmental fractionation of working memory. In: VALLAR, G.; SHALLICE, T. (Ed.) Neuropsychological Impairments of Short-Term Memory. Cambridge: Cambridge University Press, 1990. p.221-46.

HUE, C. W.; ERICKSON, J. R. Short-term memory for chinese characters and radicals. Memory and Cognition, v.16, n.3, p.196-205, 1988.

JONES, D. M.; HUGHES, R. W.; MACKEN, W. J. Perceptual organization masquerading as phonological storage: Further support for a perceptual-gestural view of short-term memory. Journal of Memory and Language, v.54, n.2, p.265-81, 2006.

. The phonological store abandoned. The Quarterly Journal Of Experimental Psychology, v.60, n.4, p.505-11, 2007.

KEMPS, E. Complexity effects in visuo-spatial working memory: implications for the role of long-term memory. Memory, v.9, n.1, p.13-27, 2001.

KEMPS, E.; ANDRADE, J. Dynamic visual noise reduces confidence in short-term memory for visual information. Cognitive Processing, v.13, n.2, p.183-8, 2012.

KLAUER, K. C.; ZHAO, Z. Double Dissociations in Visual and Spatial Short-Term Memory. Journal of Experimental Psychology, v.133, n.3, p.355-81, 2004.

KOSSLYN, S. M. Image and Brain: The Resolution of the Imagery Debate. Cambridge: Mit Press, 1994. p.516.

LIESEFELD, H. R.; ZIMMER, H. D. The advantage of mentally rotating clockwise. Brain and Cognition, v.75, n.2, p.101-10, 2011.

LOGIE, R. H. Visuo-spatial processing in working memory. The Quarterly Journal of Experimental Psychology, v.38, n.2, p.229-47, 1986.

. Visuo-Spatial Working Memory. Hove: Lawrence Erlbaum Associates Ltd., 1995. p.176. 
LOGIE, R. H. Spatial and Visual Working Memory: A Mental Workspace. In: IRWIN, D.; ROSS, B. H. (Ed.) Cognitive vision: The psychology of learning and motivation. San Diego: Academic Press, 2003. p.37-78.

. The Functional Organization and Capacity Limits of Working Memory. Current Directions in Psychological Science, v.20, n.4, p.240-5, 2011.

LOGIE, R. H.; DELLA SALA, S. Disorders of visuospatial working memory. In: SHAH, P.; MYIAKE, A. (Ed.) The Cambridge Handbook of Visuospatial Thinking. Cambridge: Cambridge University Press, 2005. p.81-120.

LOGIE, R. H. et al. Visual similarity effects in immediate verbal serial recall. The Quarterly Journal of Experimental Psychology, v.53, n.3, p.626-46, 2000.

LOGIE, R. H.; MARCHETTI, C. Visuo-spatial working memory: Visual, spatial or central executive? In: LOGIE, R. H.; DENIS, M. (Ed.) Mental images in human cognition. Amsterdam: Elsevier, 1991. p.105-15.

LOGIE, R. H.; PEARSON, D. G. The Inner Eye and the Inner Scribe of Visuo-spatial Working Memory: Evidence from Developmental Fractionation. European Journal of Cognitive Psychology, v.9, n.3, p.241-57, 1997.

LOGIE, R. H.; VAN DER MEULEN, M. Fragmenting and integrating visuospatial working memory. In: BROCKMOLE, J. R. (Ed.) The Visual World in Memory. Hove: Taylor \& Francis, 2009. p.1-32.

McCONNELL, J.; QUINN, J. G. Interference in Visual Working Memory. The Quarterly Journal of Experimental Psychology, v.53, n.1, p.53-67, 2000.

Complexity factors in visuo-spatial working memory. Memory, v.12, n.3, p.338-50, 2004.

OLSON, I. R.; MARSHUETZ, C. Remembering "what” brings along "where" in visual working memory. Perception And Psychophysics, v.67, n.2, p.185-94, 2005.

ORSINI, A. et al. Factors which influence the dificulty of the spatial path in corsi's block-tapping test. Perceptual and Motor Skills, v.92, n.3, p.732-8, 2001.

PARKER, A.; DAGNALL, N. Concreteness effects revisited: The influence of dynamic visual noise on memory for concrete and abstract words. Memory, v.17, n.4, p.397-410, 2009.

PARMENTIER, F. B. R. et al. Organization of visuo-spatial serial memory: interaction of temporal order with spatial and temporal grouping. Psychological Research, v.70, n.3, p.200-17, 2006.

PARMENTIER, F. B. R.; ELFORD, G.; MAYBERY, M. Transitional information in spatial serial memory: path characteristics affect recall performance. Journal of Experimental Psychology: Learning, Memory and Cognition, v.31, n.3, p.412-27, 2005.

PARRA, M. A. et al. Short-term memory binding deficits in Alzheimer's disease. Brain, v.132, n.4, p.1057-66, 2009.

PAZZAGLIA, F.; CORNOLDI, C. The role of distinct components of visuo-spatial working memory in the processing of texts. Memory, v.7, n.1, p.19-41, 1999.

PEARSON, D. G. Imagery and the visuo-spatial sketchpad. In: ANDRADE, J. (Ed.) Working memory in perspective. Hove: Psychology Press, 2001. p.33-59. 
PEREIRA, M. S.; GALERA, C. A integração da informação visual e espacial na memória de trabalho: evidências baseadas no efeito do ruído visual dinâmico. (em prepardo) POSTLE, B. R. et al. The selective disruption of spatial working memory by eye movements. The Quarterly Journal of Experimental Psychology, v.59, n.1, p.100-20, 2006.

QUINN, J. G. Towards a clarification of spatial processing. The Quarterly Journal of Experimental Psychology, v.47, n.2, p.465-80, 1994.

Movement and visual coding: the structure of visuo-spatial working memory. Cognitive Processing, v.9, n.1, p.35-43, 2008.

. Theories and debate in visuo-spatial working memory: the questions of access and rehearsal. In: GYSELINCK, V.; PAZZAGLIA, F. (Ed.) From Mental Imagery to Spatial Cognition and Language: Essays in Honour of Michel Denis. Hove: Psychology Press, 2012. p.106-24.

QUINN, J. G.; McCONNELL, J. Indications of the functional distinction between the components of visual working memory. Psychologische Beitrage, v.38, n.3-4, p.355-67, 1996.

The interval for interference in conscious visual imagery. Memory, v.14, n.2, p.241-52, 2006.

QUINN, J. G.; RALSTON, G. E. Movement and attention in visual working memory. The Quarterly Journal of Experimental Psychology, v.38, n.4, p.689-703, 1986.

REPOVS, G.; BADDELEY, A. D. The multi-component model of working memory: explorations in experimental cognitive psychology. Neuroscience, v.139, n.1, p.5-21, 2006.

ROSSI-ARNAUD, C.; PIERONI, L.; BADDELEY, A. D. Symmetry and binding in visuo-spatial working memory. Neuroscience, v.139, n.1, p.393-400, 2006.

SALAMÉ, P.; BADDELEY, A. D. Disruption of short-term memory by unattended speech: Implications for the structure of working memory. Journal of Verbal Learning and Verbal Behavior, v.21, n.2, p.150-64, 1982.

SANTANA, J. J. R. A. de et al. Interference of dynamic visual noise on encoding visual information in working memory. Psicologia: Reflexão e Crítica. (no prelo).

SANTANA, J. J. R. A. de; GALERA, C. Incidental encoding in the serial order of visual-spatial events in working memory: evidences based on a change detection task. Spanish Journal of Psychology. (no prelo).

SHALLICE, T. From neuropsychology to mental structure. Cambridge: Cambridge University Press, 1988. v.14, p.462.

SMYTH, M. M. Interference with spatial immediate memory in the absence of eye movements. The Quarterly Journal of Experimental Psychology, v.49A, n.4, p.940-9, 1996.

SMYTH, M. M. et al. Serial position memory in the visual-spatial domain: Reconstructing sequences of unfamiliar faces. The Quarterly Journal of Experimental Psychology, v.58, n.5, p.909-30, 2005.

SMYTH, M. M.; PENDLETON, L. R. Working memory for movements. The Quarterly Journal of Experimental Psychology, v.41, n.2, p.235-50, 1989.

SMYTH, M. M.; SCHOLEY, K. A. Interference in spatial memory. Journal of Experimental Psychology: Learning, Memory and Cognition, v.14, n.4, p.668-75, 1994. 
SMYTH, M. M.; WALLER, A. Movement imagery in rock climbing: patterns of interference from visual, spatial and kinaesthetic secondary tasks. Applied Cognitive Psychology, v.12, n.2, p.145-157, 1998.

TOMS, M.; MORRIS, N.; FOLEY, P. Characteristics of visual interference with visuospatial working memory. British Journal of Psychology, v.85, n.1, p.131-44, 1994.

TRESCH, M. C.; SINNAMON, H. M.; SEAMON, J. G. Double dissociation of spatial and object visual memory: Evidence from selective interference in intact human subjects. Neuropsychologia, v.31, n.3, p.211-19, 1993.

RESUMO - O estudo da memória de trabalho está centrado no fracionamento desse sistema em subsistemas especializados na armazenagem de informação verbal, visuoespacial e multimodal. Neste artigo abordamos o fracionamento da memória visuoespacial em seus componentes visuais e espaciais. A presença de códigos visuais na memória de trabalho é sugerida pelos efeitos de similaridade visual e de informação visual irrelevante. A memória espacial parece depender fortemente de recursos da atenção e sua relação com a memória visual ainda é controversa.

PALAVRAS-CHAVE: Memória de trabalho, Esboço visuoespacial, Memória visual, Memória espacial.

ABSTRACT - Research on working memory has been focused on fractionating specialized subsystems responsible for storage of verbal, visuospatial and multimodal information. In the present paper, we review the fractionation of visuospatial working memory into separate visual and spatial components. The availability of visual codes in working memory is supported by the effects of visual similarity and irrelevant information. Spatial memory seems to depend on attention resources and its relationship with visual memory is still controversial

KEYWORDS: Working memory, Visuospatial sketchpad, Visual memory, Spatial memory.

Cesar Galera é professor da Faculdade de Filosofia, Ciências e Letras de Ribeirão Preto, Universidade de São Paulo. @ - algalera@usp.br

Ricardo Basso Garcia é doutorando em Psicobiologia da Faculdade de Filosofia, Ciências e Letras de Ribeirão Preto, Universidade de São Paulo. @ - rbgarcia@gmail.com

Rafael Vasques é mestrando em Psicobiologia da Faculdade de Filosofia, Ciências e Letras de Ribeirão Preto, Universidade de São Paulo. @ - vasques.rf@gmail.com

Os autores agradecem ao CNPq (processo 6855/2009-7, CG), à Capes (RBG) e à Fapesp (2012/17281-0, RV).

Recebido em 21.1.2013 e aceito em 31.1.2013. 